\title{
Research
}

\section{Rapid cancer diagnosis for patients with vague symptoms:}

a cost-effectiveness study

\begin{abstract}
Background

A pilot rapid diagnosis centre (RDC) allows GPs within targeted clusters to refer adults with vague and/or non-specific symptoms suspicious of cancer, who do not meet criteria for referral under an urgent suspected cancer (USC) pathway, to a multidisciplinary RDC clinic where they are seen within 1 week.
\end{abstract}

\section{Aim}

To explore the cost-effectiveness of the RDC compared with standard clinical practice.

\section{Design and setting}

Cost-effectiveness modelling using routine data from Neath Port Talbot Hospital, Wales.

\section{Method}

Discrete-event simulation modelled a cohort of 1000 patients from referral to radiological diagnosis based on routine RDC and hospital data. Control patients were those referred to a USC pathway but then downgraded. Published sources provided estimates of patient quality of life (QoL) and pre-diagnosis anxiety. The model calculates time to diagnosis, costs, and qualityadjusted life years (QALYS), and estimates the probability of the RDC being a cost-effective strategy.

\section{Results}

The RDC reduces mean time to diagnosis from 84.2 days in usual care to 5.9 days if a diagnosis is made at clinic, or 40.8 days if further investigations are booked during RDC. $\mathrm{RDC}$ provision is the superior strategy (that is, less costly and more effectivel compared with standard clinical practice when run near or at full capacity. However, it is not cost-effective if capacity utilisation drops below $80 \%$.

\section{Conclusion}

An RDC for patients presenting with vague or non-specific symptoms suspicious of cancer in primary care reduces time to diagnosis and provides excellent value for money if run at $\geq 80 \%$ capacity.

\section{Keywords}

cancer diagnosis; cost-effectiveness; early detection of cancer; general practice; nonspecific symptoms; rapid diagnosis centre.

\section{INTRODUCTION}

A cancer diagnosis at an advanced stage worsens survival, quality of life, and patient experience. ${ }^{1}$ Furthermore, it increases healthcare costs due to more expensive treatments, higher toxicity, and symptom burden, ${ }^{2}$ and imposes a high financial burden on patients and society. Expediting cancer diagnosis and treatment initiation by improving patient navigation through complex diagnostic pathways is therefore central to recent national strategies. ${ }^{3}$ The National Institute for Health and Care Excellence (NICE) revised its guidelines to achieve timely cancer diagnosis and treatment based on 'risk thresholds' or 'red flags. ${ }^{4}$ If the risk of symptoms being caused by cancer is above a positive predictive value of $3 \%$, referral to the urgent suspected cancer pathway (USC) is warranted. However, $50 \%$ of patients with cancer in UK general practice do not present with the red flags required for referral to a site-specific suspected cancer pathway. These patients with vague and/or nonspecific symptoms wait a median of 34 days longer to diagnosis compared with patients presenting with alarm symptoms. ${ }^{5}$

Recognising that existing guidelines and pathways underserve this patient population, the Danish "three-legged model' ${ }^{\prime}$ was adapted to provide fast access to multidisciplinary diagnostic centres for patients with nonspecific and/or vague symptoms that could be due to cancer. ${ }^{7}$ In Wales, two health boards are piloting rapid diagnosis centres (RDCs), coordinated through the Welsh Cancer Network, to evaluate the feasibility, acceptability, effectiveness, and cost-effectiveness of these services through referral from primary care. The Swansea Bay University Health Board (SBUHB) pilot RDC opened in June 2017 and runs two halfday clinics a week with five available clinic slots. The current multidisciplinary team (MDT) consists of a consultant physician, a radiologist, a clinical nurse specialist (CNS), and a healthcare support worker (HCSW). Management and clinical guidance are provided by the RDC coordination manager and GP project lead. GPs can refer adults with vague and/or non-specific symptoms that could be due to cancer but do not meet criteria for referral under a USC pathway to the RDC. Appointments are usually confirmed within 48 hours of referral and chest X-ray and blood tests are initiated by the GP prior to the RDC appointment. Patients are seen at the RDC within a week and leave the clinic with either a diagnosis and management plan or further investigations booked.
B Sewell, PhD, senior lecturer in health economics; M Jones, PhD, research officer in health economics; D Fitzsimmons, PhD, professor of health outcomes research and director, Swansea Centre for Health Economics, Swansea University, Swansea, Wales. H Gray, BSc (Hons), rapid diagnosis centre coordination manager, Rapid Diagnosis Centre; C LloydBennett, RGN, BSc, rapid diagnosis centre Macmillan clinical nurse specialist, Rapid Diagnosis Centre; K Beddow, MSc, senior service manager; M Bevan, BSc, MSc, FRCP, FFMLM, unit medical director, Neath Port Talbot Hospital, Swansea Bay University Health Board, Neath, Port Talbot, Wales. H Wilkes, MRCGP, GP and Rapid Diagnosis Centre project lead, Dr Wilkes \&
Partners, Briton Ferry Health Centre, Swansea Bay University Health Board, Neath, Wales.

\section{Address for correspondence}

Bernadette Sewell, Swansea Centre for Health Economics, College of Human and Health Sciences, Swansea University, Singleton Park, Swansea SA2 8PP, Wales, UK

Email: b.diethartaswansea.ac.uk

Submitted: 26 July 2019; Editor's response: 20 August 2019; final acceptance: 22 September 2019

\section{OThe Authors}

This is the full-length article (published online 14 Jan 2020) of an abridged version published in print. Cite this version as: Br J Gen Pract 2020; DOI: https://doi.org/10.3399/bjgp20X708077 


\section{How this fits in}

Patients presenting in general practice with vague or non-specific symptoms suspicious of cancer are currently underserved. Rapid diagnosis centres (RDCs) seek to address the unmet needs of patients who do not meet referral criteria to an urgent suspected cancer pathway. However, no evaluations of the cost-effectiveness of these novel services exist. This evaluation found that a pilot RDC reduced time to diagnosis and provided excellent value for money at $>80 \%$ capacity utilisation compared with standard clinical practice.

Although the RDC addresses an important unmet need, the additional resources and costs required may displace other interventions and services for patients with cancer. This evaluation is therefore aimed at exploring the cost-effectiveness of the RDC in its first year of operation compared with standard clinical practice, to ensure that the $\mathrm{RDC}$ is doing more good than harm.

\section{METHOD}

Patient-level discrete-event simulation (DES) and decision analytic modelling were used to estimate the time from referral to diagnosis and the cost-effectiveness of the RDC compared with standard clinical practice.

DES models a sequence of events, following patients as they move through the cancer pathway. It allows resource costs and health outcomes to be modelled and is utilised widely in healthcare settings to tackle issues relating to capacity ${ }^{9}$ and pathway redesign. 8,10

The model was implemented in Microsoft Excel (2016), Visual Basic for Applications (version 7.01), and SIMUL8 Professional. Deterministic and probabilistic sensitivity analyses (PSA) explored the impact of assumptions and uncertainties.

The RDC clinicians validated the modelled patient pathways and all assumptions. The evaluation followed the NICE reference case $^{11}$ and adhered to Consolidated Health Economic Evaluation Reporting Standards (CHEERS). ${ }^{12}$ An NHS and Personal Social Services perspective was adopted, following patients from referral to radiological diagnosis. The model ran over 1 year and findings were expressed as costs (in GBP at 2017 exchange rates) and quality-adjusted life years (QALYS). No discounting was applied as the time horizon did not exceed 12 months. The time horizon of 1 year was chosen to reflect the length of the available pilot data of the RDC.

\section{Study population}

Adults aged $\geq 18$ years in the pilot area who were referred by their GP to the RDC at Neath Port Talbot Hospital (NPTH) for further investigation of non-specific and/or vague symptoms that could be due to cancer between June 2017 and May 2018 were included in the evaluation.

A cohort of 1000 patients was simulated based on real-life data of the NPTH RDC (for the intervention group) and routinely collected records of outcome-matched patients within SBUHB, who were referred to the USC pathway by their GP but then downgraded to the non-urgent pathway because of the absence of red-flag symptoms (for the control group). This sample size of simulated patients was chosen because it was sufficiently large to account for the variability inherent in the data, yet not so large that the model run-time became excessive. The number was validated by comparing the key modelling summary statistics for varying patient numbers la facility that is available on SIMUL8). Data received for analysis were fully anonymised with all patient identifiers removed. Relevant permissions were in place before data release and data matching for the control group was performed by SBUHB.

\section{Modelled patient pathways}

On referral by their GP, patients attended the next available RDC clinic slot (Figure 1). Clinics began with an MDT meeting where current and available historical patient notes were discussed. Thereafter, patients saw the physician/CNS/HCSW for consultation and physical examination followed by a computed tomography (CT) scan. Alternatively, patients could start with a CT scan, followed by their physician/CNS/HCSW appointment. At the second MDT meeting all patient findings were discussed and the patients were placed into one of four categories:

- cancer diagnosis with referral to specialist;

- non-cancer diagnosis;

- no serious pathology found with discharge to GP; and

- no diagnosis; continue investigations.

Patients with suspected cancer were seen again by the CNS for a 20-minute appointment to discuss their diagnosis and begin a holistic needs assessment. Patients with non-cancer diagnoses or those who required further investigations were referred to relevant hospital departments. Patients with no serious pathology were reassured and discharged to their GP. 


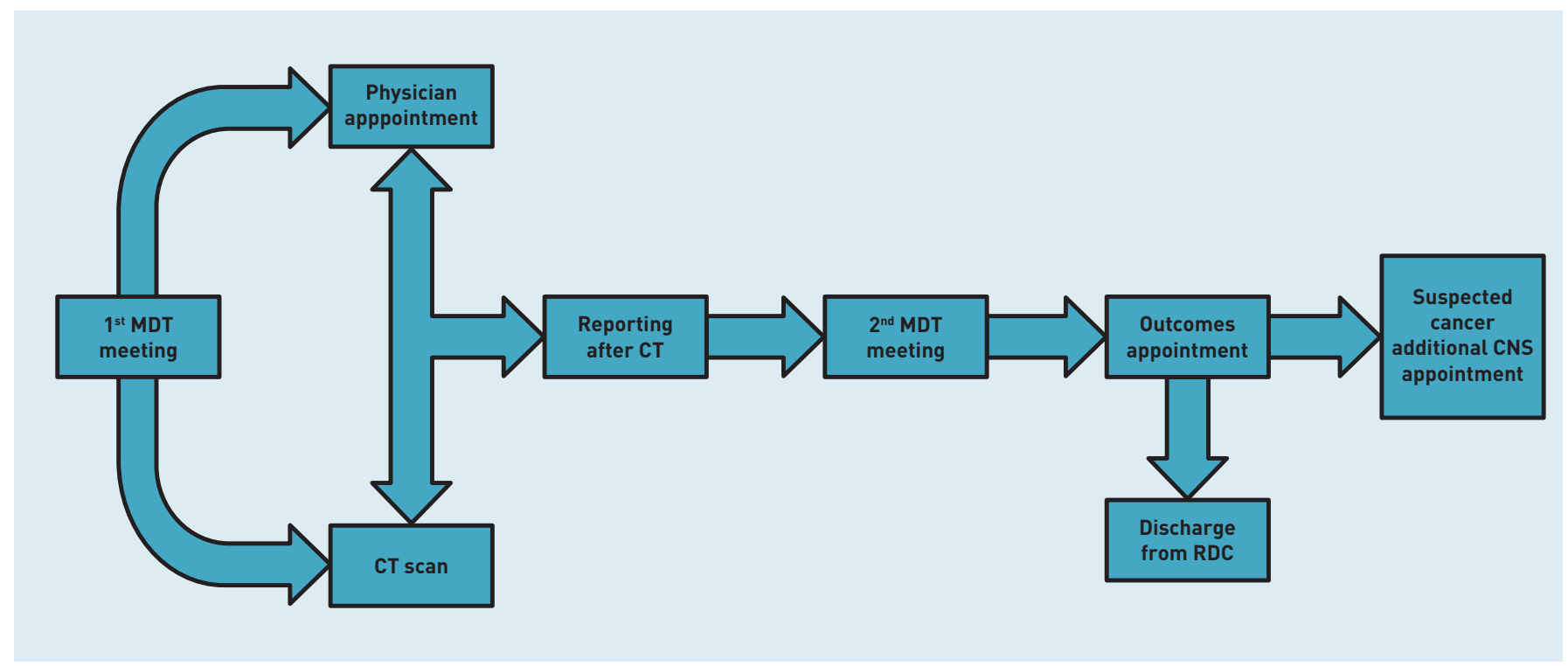

Figure 1. Schematic of the patient pathway through the rapid diagnosis centre (RDC) in the model intervention group. CNS = cancer nurse specialist. $C T=$ computed tomography. $M D T=$ multidisciplinary team.

\section{Input parameters and data sources}

Routinely collected, fully anonymised service data from the RDC provided model data inputs. The probability of a patient receiving a given diagnosis (for both RDC and control groups) was obtained from individually analysed RDC data. Expert opinion and clinical judgement were used where necessary to accurately interpret the diagnostic codes. Number of days between referral (to either RDC or USC) and diagnosis was obtained from RDC and hospital records. Distributions were fitted to the available data to allow sampling for each simulated individual in the model. Healthcare resource use was collected routinely for RDC patients and NPTH records were manually searched for control patients. RDC implementation costs included staff costs and overheads, and access to endoscopy, stationary, postage, consumables, and other non-pay items provided by the NPTH Finance Department. The number of CT scans lincluding the number of body areas scanned for each patient) and other investigative tests were recorded routinely and costed using published unit costs. ${ }^{13}$ Hospital records were searched for patients who required further investigations to identify secondary care resource use between RDC attendance and diagnosis. Similarly, control patient healthcare resource use was obtained from hospital records. Secondary care resource use was then micro-costed for each patient individually using standard unit costs. ${ }^{13}$ Primary care resource use for both model groups was established by examining the GP-reported mean frequency of GP visits in the 6 months prior to RDC referral, costed using standard unit costs. ${ }^{14}$ No utility data were recorded during the RDC. Therefore, a rapid literature review was conducted and MEDLINE, PubMed, and NHS Economic Evaluation Database were searched following a systematic review framework. Search terms included 'suspected cancer diagnosis', 'quality of life pre-diagnosis and postdiagnosis', 'anxiety', and 'vague symptoms' Ithe complete list of search terms lor search strategyl can be obtained from the authors on request).

The initial search yielded 2438 results, of which 2426 were not relevant for patients with vague symptoms, based on a scan of the abstracts. Of the remaining 12 papers, three were applicable to the evaluation in question and reported data on QoL that could be used to extrapolate utility values. ${ }^{15-17}$ However, none of these papers reported utilities based on responses to the EQ-5D questionnaire, which is the preferred patient-reported outcome measure used to calculate utilities required for health economic evaluation. ${ }^{11}$

Because of this lack of EQ-5D results in the published literature, utilities were extrapolated from the general population ${ }^{18}$ with pre-diagnosis anxiety-related decrements applied based on relative literature values. ${ }^{15-17}$ Further information and a detailed list of input parameters and data sources are available from the authors on request.

\section{Analyses}

A model-based cost-utility analysis (CUA) of the actual RDC provision between June 2017 and May 2018 assessed the incremental costs per QALY gained as a result of the introduction of the RDC compared with standard clinical practice. QALYS incorporate quantity of life and quality of life in one measure. The 


\section{Table 1. Total healthcare cost between referral and diagnosis}

\begin{tabular}{|c|c|c|c|}
\hline Outcome category & $\begin{array}{l}\text { Mean cost per RDC patient, } \\
\qquad \in \text { (SD), } n=189\end{array}$ & $\begin{array}{l}\text { Cost per control patient, } \\
\qquad \in \text { (SD), } n=85\end{array}$ & Difference, $€$ \\
\hline \multicolumn{4}{|l|}{1 patient per clinic } \\
\hline Cancer diagnosis & 2758 & 2397 (2107) & 361 \\
\hline Other diagnosis & 2758 & 871 (688) & 1887 \\
\hline No serious pathology found (discharge) & 2758 & 515 (139) & 2243 \\
\hline No diagnosis (further investigations) & $3148(214)$ & $953(381)$ & 2195 \\
\hline \multicolumn{4}{|l|}{2 patients per clinic } \\
\hline Cancer diagnosis & 1438 & 2397 (2107) & -959 \\
\hline Other diagnosis & 1438 & 871 (688) & 567 \\
\hline No serious pathology found (discharge) & 1438 & 515 (139) & 923 \\
\hline No diagnosis (further investigations) & $1828(214)$ & 953 (381) & 875 \\
\hline \multicolumn{4}{|l|}{2.78 patients per clinic ${ }^{a}$} \\
\hline Cancer diagnosis & 1068 & 2397 (2107) & -1329 \\
\hline Other diagnosis & 1068 & 871 (688) & 197 \\
\hline No serious pathology found (discharge) & 1068 & 515 (139) & 553 \\
\hline No diagnosis (further investigations) & $1458(214)$ & 953 (381) & 505 \\
\hline \multicolumn{4}{|l|}{3 patients per clinic } \\
\hline Cancer diagnosis & 998 & 2397 (2107) & -1399 \\
\hline Other diagnosis & 998 & 871 (688) & 127 \\
\hline No serious pathology found (discharge) & 998 & 515 (139) & 483 \\
\hline No diagnosis (further investigations) & $1388(214)$ & $953(381)$ & 435 \\
\hline \multicolumn{4}{|l|}{4 patients per clinic } \\
\hline Cancer diagnosis & 778 & 2397 (2107) & -1619 \\
\hline Other diagnosis & 778 & 871 (688) & -93 \\
\hline No serious pathology found (discharge) & 778 & 515 (139) & 263 \\
\hline No diagnosis (further investigations) & $1168(214)$ & 953 (381) & 215 \\
\hline \multicolumn{4}{|l|}{5 patients per clinic } \\
\hline Cancer diagnosis & 646 & 2397 (2107) & -1751 \\
\hline Other diagnosis & 646 & 871 (688) & -225 \\
\hline No serious pathology found (discharge) & 646 & 515 (139) & 131 \\
\hline No diagnosis (further investigations) & $1036(214)$ & 953 (381) & 83 \\
\hline
\end{tabular}

${ }^{2}$ Actual mean number of patients seen between June 2017 and May 2018 per clinic. RDC = rapid diagnosis centre. $S D=$ standard deviation

\section{Table 2. Summary of the results of the cost-utility analysis for a} model cohort of patients, $N=1000$

\begin{tabular}{|c|c|c|c|}
\hline & No RDC & RDC & Difference $(95 \% \mathrm{Cl})$ \\
\hline \multicolumn{4}{|l|}{2.78 patients per clinic } \\
\hline Cost & $£ 934156$ & €1 207651 & $£ 273494$ (-£445 641 to $€ 747343$ ) \\
\hline QALY & 1416 & 1425 & 9.20 (-204 to 201) \\
\hline ICER (cost per QALY gained) & $£ 29732$ & & \\
\hline \multicolumn{4}{|l|}{4 patients per clinic } \\
\hline Cost & $€ 934156$ & €917 826 & -£16 330 (-£713 924 to $€ 448358)$ \\
\hline QALY & 1416 & 1425 & 9.20 (-204 to 201) \\
\hline ICER (cost per QALY gained) & -£1775 (outperforms) & & \\
\hline \multicolumn{4}{|l|}{5 patients per clinic } \\
\hline Cost & $€ 934156$ & $€ 785836$ & -£148 320 (-£839 771 to $€ 312218$ ) \\
\hline QALY & 1416 & 1425 & $9.20(-204$ to 201$)$ \\
\hline ICER (cost per QALY gained) & $-£ 16124$ (outperforms) & & \\
\hline
\end{tabular}

ICER = incremental cost-effectiveness ratio. $Q A L Y=$ quality-adjusted life year. $R D C=$ rapid diagnosis centre incremental cost-effectiveness ratio (ICER) resulting from the CUA was compared with the willingness-to-pay threshold of $€ 20000$ per QALY gained as standardised by NICE."1 A series of deterministic one-way sensitivity analyses investigated the impact of changes in RDC costs, number of patients per clinic, healthcare costs, and alternative utility assumptions on the results. PSA calculated the probability of the intervention being cost-effective at different willingness-to-pay thresholds based on 1000 bootstrapping iterations drawn from distributions around the mean base case values, and these probabilities were presented as a costeffectiveness acceptability curve.

\section{RESULTS}

Between June 2017 and May 2018, 189 patients attended the RDC. Of these patients, $46 \%$ were male and the mean age was 70 years (standard deviation [SD] $=12.9$ years; minimum $=26$; maximum $=95$ ). Most patients presented with unexplained weight loss, pain, fatigue, and shortness of breath. The final outcomes were categorised into four groups: cancer diagnosis with referral to specialist ( $n=23,12 \%)$, non-cancer diagnosis ( $n=30,16 \%$ ), no serious pathology found with discharge to GP ( $n=68,36 \%)$, and no diagnosis; continue investigations $(n=68$, $36 \%$ ).

\section{Time to diagnosis}

Mean time to diagnosis was 5.9 days $(S D=3.4$ : minimum $=1.0 ;$ maximum $=15.0$ ) for $\mathrm{RDC}$ patients who had a cancer diagnosis, other diagnosis, or were discharged. For patients requiring further investigations, the mean time to diagnosis was 40.8 days $(S D=28.0$; minimum $=0.0$; maximum $=132.0$ ). If outliers were removed (that is, 3-month delays for four patients needing specialised services), this decreased to 33.9 days. Control patients waited 84.2 days $(S D=65.3$; minimum $=0.0$; maximum $=657.0$ ) for diagnosis .

\section{Costs between referral and diagnosis}

Total staff costs per half-day clinic were calculated as $€ 2640$ with CT scan and other test costs amounting to $€ 118.21$ per patient. Healthcare costs accumulated between RDC referral or USC downgrade and radiological diagnosis were estimated for all outcome categories in both model groups. Patients diagnosed with cancer or other conditions and discharged to their GP with no serious pathology found were assumed to only incur RDC costs. Any further investigations and all control pathways were costed individually. Costs are displayed in Table 1 for different 


\section{Table 3. Summary of one-way sensitivity analyses conducted and optimal strategy based on WTP of $€ 20000$}

\begin{tabular}{|c|c|c|}
\hline Parameter & Change & Optimal strategy \\
\hline Cost of RDC & $\begin{array}{l}\text { Change number of patients per day } \\
\text { from } 2.78 \text { to } 5\end{array}$ & RDC (outperforms) \\
\hline Cost of RDC & $\begin{array}{l}\text { Change number of patients per day } \\
\text { from } 2.78 \text { to } 4\end{array}$ & RDC (outperforms) \\
\hline Cost of RDC & $\begin{array}{l}\text { Change number of patients per day } \\
\text { from } 2.78 \text { to } 3\end{array}$ & No RDC (ICER = €22 140) \\
\hline Cost of RDC & $\begin{array}{l}\text { Change number of patients per day } \\
\text { from } 2.78 \text { to } 2\end{array}$ & No RDC (ICER = £69969) \\
\hline Cost of RDC & $\begin{array}{l}\text { Change number of patients per day } \\
\text { from } 2.78 \text { to } 1\end{array}$ & No RDC (ICER = £213 459) \\
\hline All costs & Add $20 \%$ to all costs & No RDC (ICER = €35 678) \\
\hline All costs & Subtract $20 \%$ from all costs & No RDC (ICER = €23 786) \\
\hline All costs & Add $50 \%$ to all costs & No RDC (ICER = €44 598) \\
\hline All costs & Subtract $50 \%$ from all costs & RDC (ICER = £14 866) \\
\hline $\begin{array}{l}\text { Adjusted OR for control } \\
\text { group cancer patients }\end{array}$ & $\begin{array}{l}\text { After } 2 \text { months increase cancer QALY OR } \\
\text { from } 0.69 \text { to } 0.95\end{array}$ & No RDC (ICER = £34 848) \\
\hline Cancer diagnosis utility & Utility decreases by $3 \%$ to 0.602 & No RDC (ICER = £62 637) \\
\hline Utility & $\begin{array}{l}\text { Keep all utilities } 0.664 \text { rather than } 0.62 \text { for } \\
\text { all patients until diagnosis }\end{array}$ & No RDC (ICER = £245 102) \\
\hline Utility & $\begin{array}{l}\text { Keep all utilities } 0.664 \text { rather than } 0.62 \text { for } \\
\text { all patients until diagnosis with number of } \\
\text { patients } 5 \text { per day }\end{array}$ & RDC (outperforms) \\
\hline Utility & $\begin{array}{l}\text { Keep all utilities } 0.664 \text { rather than } 0.62 \text { for } \\
\text { all patients until diagnosis with number of } \\
\text { patients } 4 \text { per day }\end{array}$ & RDC (outperforms) \\
\hline Utility & $\begin{array}{l}\text { Keep all utilities } 0.664 \text { rather than } 0.62 \text { for } \\
\text { all patients until diagnosis with number of } \\
\text { patients } 3 \text { per day }\end{array}$ & No RDC (£182 514) \\
\hline Utility & $\begin{array}{l}\text { Keep all utilities } 0.664 \text { rather than } 0.62 \text { for } \\
\text { all patients until diagnosis with number of } \\
\text { patients } 2 \text { per day }\end{array}$ & No RDC (ICER = £576 811) \\
\hline Utility & $\begin{array}{l}\text { Keep all utilities } 0.664 \text { rather than } 0.62 \text { for } \\
\text { all patients until diagnosis with number of } \\
\text { patients } 1 \text { per day }\end{array}$ & No RDC (ICER = £1 759 700) \\
\hline
\end{tabular}

Figure 2. Cost-effectiveness acceptability curve summarising results of probabilistic sensitivity analysis across 1000 runs assuming 2.78 patients per clinic at different WTP thresholds. WTP= willingness-to-pay. patient numbers per clinic to illustrate the effect of capacity on RDC per-patient costs.

\section{Cost-effectiveness}

The model base case used actual outcome data and costs of the RDC between June 2017 and May 2018 with a mean number of 2.78 patients per clinic. During this service start-up phase, the RDC was more costly and more effective compared with referral to USC pathway followed by downgrade with an ICER of $€ 29732$ (Table 2). Since July 2018, the RDC has run near or at full capacity, consistently seeing between four and five patients per clinic, with a mean number of 4.65 patients (93\% use of capacity) per clinic over the last year, thus outperforming usual care (that is, being less costly and more effective).

During deterministic sensitivity analysis (Table 3), findings were robust to changes in utility values and the assumption that the pre-diagnosis stage is associated with increased anxiety. Findings are sensitive to changes in costs and the number of patients attending each clinic. As such, due to economies of scale, the RDC outperforms standard clinical practice with four or five patients per clinic, but is not cost-effective when only one, two, or three patients are seen.

PSA based on the observed 2.78 patients per clinic in the RDC start-up phase produced a probability of being cost-effective of $48.5 \%$ at a willingness-to-pay threshold of $€ 20000$ (Figure 2). At maximum capacity of five patients per clinic, RDC outperforms standard clinical practice with a 56.0\% probability of being cost-effective.

\section{DISCUSSION}

\section{Summary}

A pilot RDC for patients with vague and/or non-specific symptoms suspicious of cancer is cost-effective compared with standard clinical practice available in Wales (that is, referral to USC pathway and subsequent downgrade due to the absence of red-flag symptoms) when run near or at full capacity.

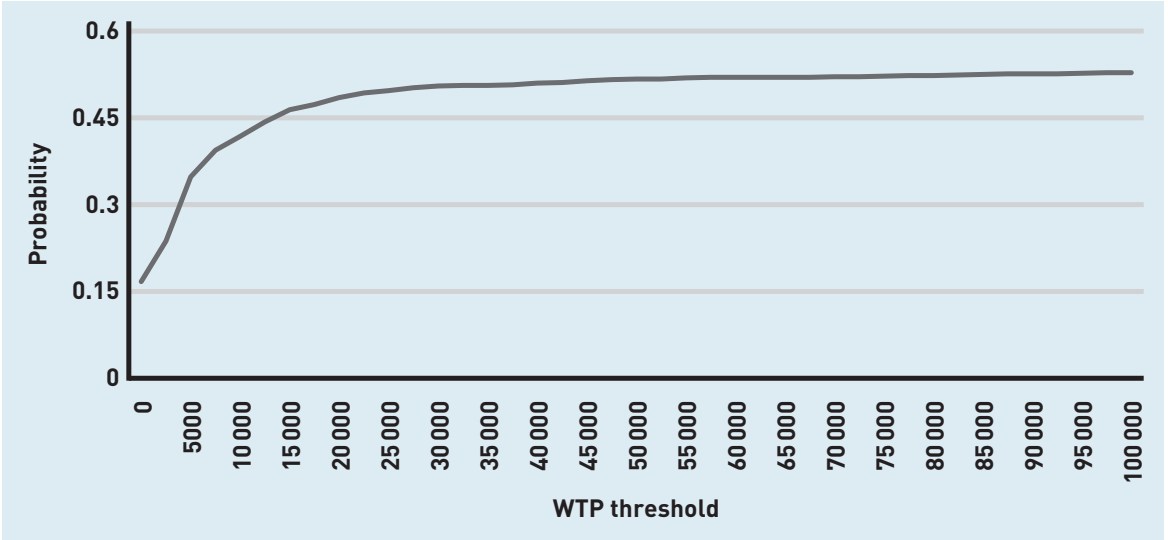


At between $80 \%$ and $100 \%$ capacity, the RDC produces more QALYs and is less costly, and thus outperforms standard clinical practice. Below $80 \%$ capacity, the RDC is not costeffective at a £20 000 willingness-to-pay threshold. Mean time to diagnosis was 84.2 days $(S D=65.3$ ) in the control group. This was reduced to 5.9 days (SD $=3.4$ ) in patients who were diagnosed directly at the $\mathrm{RDC}$ clinic and to 40.8 days (SD $=30.0$ ) if further investigations following RDC were warranted.

\section{Strengths and limitations}

This analysis is the first to consider the cost-effectiveness of an RDC for patients with vague and/or non-specific symptoms suspicious of cancer in the UK, using a relevant control, routine data, and DES. DES has proven valid and useful in various healthcare settings to model the impact of patient pathway changes, ${ }^{8}$ redesign, ${ }^{10}$ and capacity changes. ${ }^{9}$ However, the complexity of the service, patient population, and case mix required simplifications and assumptions to be made. These were verified by RDC key team members. The analysis assumes that all RDC staff are present throughout, even if the clinic is not at full capacity. As this may not accurately reflect routine practice, it could overestimate RDC implementation cost and underestimate cost-effectiveness at lower capacities. Detailed information was available for the patient pathway after RDC attendance. However, it was impossible to track patients in the control group who required further investigations after their first outpatient appointment for which no further patient notes were accessible. Therefore, the time to diagnosis was assumed to be the time to first outpatient appointment, which may underestimate time to diagnosis in a few cases. Although the impact of this omission is likely small, these results may still represent a conservative estimate of the cost-effectiveness of the RDC.

Furthermore, QoL data were not collected routinely as part of the RDC process. Utilities had to be extrapolated from the general population ${ }^{18}$ with decrements applied based on relative literature values. ${ }^{15-17}$ Although this was deemed the best available approach, this amount of data manipulation may have introduced bias. However, removing the effect of anxiety on patient QoL in sensitivity analysis showed that the effect of this uncertainty was small and did not change the direction of results.

Data used to populate the model were taken from the first year of the RDC pilot operation. These immature data did not allow extrapolation of the longer-term impact of the RDC on healthcare resource use, patient outcomes, or survival. Considering the potential positive effect of earlier diagnosis on prognosis, this omission will underestimate the cost-effectiveness of the RDC. It is therefore planned to extend the model to a longer-term time horizon as soon as more mature data become available.

\section{Comparison with existing literature}

Although evaluations of rapid and multidisciplinary diagnosis centres are currently underway, ${ }^{19}$ this is the first robust and complete analysis of the costeffectiveness of the RDC as part of routine care in the UK. Also, to the best of the authors' knowledge, no economic evaluations of the Danish cancer patient pathway for patients presenting with non-specific symptoms and signs of cancer (NSSC-CPP), on which the UK model of this service is based, exist.

Considering clinical outcomes, the cancer diagnosis rate of $12 \%$ in the RDC was comparable with conversion rates in many site-specific cancer pathways. Urgent suspected cancer pathway conversion rates were reported to be between a median of $8.0 \%$ and $17.0 \%,{ }^{20}$ and to have a mean of $11.8 \%,{ }^{21}$ depending on the use of urgent pathways, referral rates, and cancer type. Also, a recent systematic review and metaanalysis of fast-track diagnosis of colorectal cancer found a cancer detection rate of $7.7 \%$ (confidence interval $=6.9 \%$ to $8.5 \%$ ). ${ }^{22}$ Furthermore, the current study's results are similar to data from Danish NSSC-CPP patients ( $n=23$ 934), which suggest a mean cancer diagnosis rate in an RDC setting of $11 \%$ (with values between $9 \%$ and $12 \%$ ). ${ }^{23}$

\section{Implications for research and practice}

Referral to rapid diagnosis services from primary care for patients with vague and/ or non-specific symptoms suspicious of cancer addresses an important unmet need and provides value for money when run near or at full capacity. Furthermore, it reduces time to diagnosis and has the potential to improve patient outcomes. Development of a minimum dataset or registry, including collection of patient QoL data, should be considered to aid any future evaluations of the clinical and cost-effectiveness of the RDC. Harmonisation of key data items of RDC models across the UK and consensus on a suitable control in local and national contexts will be crucial in any comparisons. Using this first model as a template will enable further investigation as data mature.
Contribute and read comments about this article: bjgp.org/letters 


\section{REFERENCES}

1. Silver JK, Raj VS, Fu JB, et al. Cancer rehabilitation and palliative care: critical components in the delivery of high-quality oncology services. Support Care Cancer 2015; 23(12): 3633-3643.

2. Laudicella M, Walsh B, Burns E, et al. Cost of care for cancer patients in England: evidence from population-based patient-level data. Br J Cancer 2016; 114(11): 1286-1292

3. Welsh Cancer Network. Cancer delivery plan for Wales 2016-2020. http://umw walescanet.wales.nhs.uk/sitesplus/documents/1113/Cancer\%20Delivery\%20 Plan\%202016-2020.pdf laccessed 1 Dec 2019].

4. National Institute for Health and Care Excellence. Suspected cancer: recognition and referral. NG12. 2017. https://www.nice.org.uk/guidance/ng12 laccessed 1 Dec 2019).

5. Neal RD, Din NU, Hamilton W, et al. Comparison of cancer diagnostic intervals before and after implementation of NICE guidelines: analysis of data from the UK General Practice Research Database. Br J Cancer 2014; 110(3): 584-592.

6. Vedsted $\mathrm{P}$, Olesen F. A differentiated approach to referrals from general practice to support early cancer diagnosis - the Danish three-legged strategy. $\mathrm{Br} J$ Cancer 2015; 112(Suppl 1): S65-S69.

7. Fuller E, Fitzgerald K, Hiom S. Accelerate, Coordinate, Evaluate Programme - a new approach to cancer diagnosis. Br J Gen Pract 2016; DOI: https://doi. org/10.3399/bjgp16X684457.

8. Katsaliaki K, Mustafee N. Applications of simulation within the healthcare context. J Oper Res Soc 2011; 62(8): 1431-1451.

9. Devapriya P, Strömblad CT, Bailey MD, et al. StratBAM: a discrete-event simulation model to support strategic hospital bed capacity decisions. J Med Syst 2015; 39(10): 130

10. Rachuba S, Salmon A, Zhelev Z, et al. Redesigning the diagnostic pathway for chest pain patients in emergency departments. Health Care Manag Sci2018; 21(2): 177-191.

11. National Institute for Health and Care Excellence. Process and methods. In: Developing NICE quidelines: the manual. PMG20. 2017. https://www.nice.org. uk/process/pmg20/chapter/introduction-and-overview laccessed 1 Dec 2019).
12. Husereau D, Drummond M, Petrou S, et al. Consolidated Health Economic Evaluation Reporting Standards (CHEERS) statement. Int J Technol Assess Health Care 2013; 29(2): 117-122.

13. NHS Improvement. NHS reference costs 2016 to 2017. https://improvement nhs.uk/resources/reference-costs/ laccessed 1 Dec 2019).

14. Curtis L, Burns A. Unit costs of health and social care 2017. 2017. https://mww. pssru.ac.uk/project-pages/unit-costs/unit-costs-2017/ laccessed 1 Dec 2019).

15. Moseholm E, Rydahl-Hansen S, Lindhardt BØ. Undergoing diagnostic evaluation for possible cancer affects the health-related quality of life in patients presenting with non-specific symptoms. PloS One 2016; 11(2): e0148463.

16. Moseholm E, Rydahl-Hansen S, Overgaard D, et al. Health-related quality of life, anxiety and depression in the diagnostic phase of suspected cancer, and the influence of diagnosis. Health Qual Life Outcomes 2016; 14: 80.

17. Walming S, Block M, Bock D, et al. Timely access to care in the treatment of rectal cancer and the effect on quality of life. Colorectal Dis 2018; 20(2): 126-133.

18. Janssen MF, Szende A, Cabases JM, et al. Population norms for the EQ-5D-3L: a cross-country analysis of population surveys for 20 countries. Eur $\mathrm{J}$ Health Econ 2019; 20(2): 205-216.

19. Nicholson BD, Oke J, Friedemann Smith C, et al. The Suspected CANcer (SCAN) pathway: protocol for evaluating a new standard of care for patients with non-specific symptoms of cancer. BMJ Open 2018; 8(1): e018168.

20. Møller H, Gildea C, Meechan D, et al. Use of the English urgent referral pathway for suspected cancer and mortality in patients with cancer: cohort study. BMJ 2015; 351: h5102.

21. Douglas CM, Carswell V, Montgomery J. Outcomes of urgent suspicion of head and neck cancer referrals in Glasgow. Ann R Coll Surg Engl 2019; 101(2): 103-106.

22. Mozdiak E, Weldeselassie Y, McFarlane M, et al. Systematic review with metaanalysis of over 90000 patients. Does fast-track review diagnose colorectal cancer earlier? Ailment Pharmacol Ther 2019; 50(4): 348-372.

23. Moseholm E, Lindhardt $B \emptyset$. Patient characteristics and cancer prevalence in the Danish cancer patient pathway for patients with serious non-specific symptoms and signs of cancer - a nationwide, population-based cohort study. Cancer Epidemiol 2017; 50(Pt A): 166-172. 